presence of upper GI symptoms; however, further work is needed to evaluate the prevalence of upper GI symptoms in this population.

Disclosure of Interest None Declared.

\section{PTH-142 THE ROLE OF SEHCAT SCANNING IN PATIENTS WITH CHRONIC DIARRHOEA: RESULTS FROM A NEW SERVICE}

doi:10.1136/gutjnl-2013-304907.629

1."G H Bain, ${ }^{2} \mathrm{~F}$ McKiddie, ${ }^{2} \mathrm{~L}$ Lovell, ${ }^{2} \mathrm{H}$ Gemmell, ${ }^{1} \mathrm{~A}$ W McKinlay, ${ }^{1} \mathrm{~J}$ S Leeds. 'Gastroenterology; ${ }^{2}$ Nuclear Medicine, Aberdeen Royal Infirmary, Aberdeen, UK

Introduction Chronic diarrhoea is a common reason for referral to gastroenterology departments and often multiple investigations are undertaken. Bile acid malabsorption is an under recognised cause of chronic diarrhoea and currently occupies a lower tier in the investigatory pathway. SeHCaT scanning has been available in our region for the last 2 years and therefore the aim of this study was to investigate the role that this test has in such patients.

Methods All patients referred for a SeHCaT scan were identified by searching by procedure in the Nuclear Medicine department. Patient demographics, indication, number of previous tests, surgical history and SeHCaT result were noted. The cut off for an abnormal test was $<15 \%$ retention at 7 days. Notes were reviewed to determine which patients had treatment and the response rate. In those with a negative result, the final diagnosis (if known) was recorded. Results 122 patients (95 female, median age 50 years) had undergone a SeHCaT scan for investigation of chronic diarrhoea during the period January 2011 to July 2012. 61/122 (50\%) patients had a SeHCaT retention $<15 \%$ with 30 having retention values $<5 \%, 19$ between 5.1 - 10\% and 12 between 10.1 - 15\%. An abnormal SeHCaT scan was associated with previous bowel surgery (Odds ratio $14.2,95 \%$ CI 1.8-113.1, $\mathrm{p}=0.002$ ) but not gender (odds ratio $2.095 \%$ CI $0.8-4.7, \mathrm{p}=\mathrm{ns}$ ) or previous cholecystectomy (odds ratio $1.295 \%$ CI $0.5-2.7, \mathrm{p}=\mathrm{ns}) .45 / 53(84.9 \%)$ patients were commenced on bile acid sequestrants (mainly cholestyramine) with a good response to treatment. 13 patients were intolerant of cholestyramine and switched to colesevalam of which 10 have so far had clinical improvement. Prior to SeHCat scanning patients had undergone a median of 2.5 other investigations (range $0-9$ ). Final diagnosis was bile acid diarrhoea $(\mathrm{n}=61)$, irritable bowel syndrome $(n=34)$, malabsorption $(n=3)$, Crohns disease $(n=2)$, coeliac disease $(n=1)$, diverticular disease $(n=1)$, small bowel bacterial overgrowth $(n=1)$ and still being investigated $(n=19)$.

Conclusion In patients with chronic diarrhoea, SeHCaT scanning has a high yield and is associated with good clinical response to treatment with cholestyramine. We did not find that previous cholecystectomy was a risk factor but confirm that bowel resection appears to be. Switching to colesevalam is effective when cholestyramine is not tolerated.

Disclosure of Interest None Declared.

\section{PTH-143 CYTOSPONGE INSTEAD OF ENDOSCOPY IN SYMPTOMATIC PATIENTS: A FEASIBILITY STUDY}

doi:10.1136/gutjnl-2013-304907.630

1."H Saravanapavan, 'J M Hoare. 'Department of Gastroenterology, St Mary's Hospital, Imperial College NHS Trust, London, UK

Introduction The estimated annual incidence for oesophageal adenocarcinoma in individuals with Barrett's oesophagus is 0.2$0.5 \%{ }^{1}$. However, endoscopic screening of individuals with risk factors for Barrett's oesophagus including chronic heartburn and reflux $^{2}$ are not part of current clinical recommendations. The cytosponge, a non-endoscopic immunocytological screening kit is undergoing a multicentre study and promises to alter our current practice $^{3}$. The cytosponge is less invasive than gastroscopy and would reduce the burden on endoscopy units if screening is to be introduced. It has also been shown to detect other benign diseases such as helicobacter, oesophagitis and candidiasis ${ }^{4}$ and therefore may be able to replace routine gastroscopy in symptomatic patients. The aim of this study was to examine the percentage of endoscopy referrals to St Marys hospital that could be alternatively investigated with a non-endoscopic sampling method such as the cytosponge to detect Barrett's oesophagus and/or exclude more serious pathology.

Methods All pending endoscopy requests were audited on a randomly selected day. Of these, gastroscopy referrals from GP practises and outpatient department were analysed. Patients who were suitable for the cytosponge were identified using the following criteria. Inclusion - Age 45 years and above, symptoms of dyspepsia and reflux; Exclusion - Previous diagnosis of Barrett's oesophagus, previous endoscopy in the last year, portal hypertension, patients on clopidogrel or warfarin, clotting disorders.

Results A total of 161 gastroscopy referral forms were analysed; $73 \%$ from outpatients and $27 \%$ from GP surgeries. $22 \%$ of referrals were for dyspepsia and $8 \%$ for reflux. $16 \%$ of referrals were suitable for cytosponge as defined by the inclusion and exclusion criteria.

Conclusion One in six referrals from GP surgeries and outpatients could be offered cytosponge instead of endoscopy for detection of Barrett's oesophagus. Cytosponge would reduce cost, enable rapid bedside testing and provide a non-invasive method for individuals reluctant to have an endoscopic procedure. It could also be extended to detect benign oesophageal pathology and therefore avoid secondary care referrals and waiting list pressures.

Disclosure of Interest None Declared.

\section{REFERENCES}

1. Yousef $\mathrm{F}$ et al. The incidence of esophageal cancer and high-grade dysplasia in Barrett's oesophagus: a systematic review and meta-analysis. Am J Epidemiol. 2008: 168:237-49

2. Guidelines for the diagnosis and management of Barrett's columnar-lined oesophagus. BSG. 2005

3. Kadri SR et al. Acceptability and accuracy of a non-endoscopic screening test for Barrett's oesophagus in primary care: cohort study. BMJ. 2010: Sep 10: 341:c4372

4. O'Donovan M, Lao-Sirieix P and Fitzgerald RC, Non-endoscopic diagnostic tests for esophageal diseases and H.pylori using the Cytosponge, Gastroenterology. 2012: Vol. 142, Issue 5, Supplement 1, Page S-421

\section{PTH-144 BOWEL SCREENING IN WELSH PRISONS}

doi:10.1136/gutjnl-2013-304907.631

1."H Heard, 'S Darwin. 'Bowel Screening UK, Llantrisant, UK

Introduction The aim of the bowel screening programme in Wales is to reduce mortality from bowel cancer by $15 \%$ in the population invited for screening by 2020 . Some groups of the population are difficult to reach, particularly if they are not registered with a General Practitioner (G.P.). Not all prisoners are registered with a G.P. and a task and finish group was established to develop strategies to identify and invite prisoners for screening.

Methods Contact was made with governors and health care staff at the five prisons in Wales and an education programme provided for prison staff. Data sharing agreements were developed and agreed for each prison. Protocols and care pathways were developed for screening prisoners comprising of a slightly modified service model to the standard bowel screening programme. A pilot was established in 2 prisons and the service model modified in response to issues.

It was agreed that contact with prisoners would be through health care staff. There are around 110 prisoners within the eligible age range in Welsh prisons and during the pilot prison healthcare staff notified BSW of eligible prisoners. Invitations and test kits were sent in batches to the prisoner via the medical centre where staff arranged for prisoners to be offered the opportunity to participate. 